\title{
LARGE FILLING-FACTOR-DEPENDENT SPIN SPLITTING IN MAGNETOOPTIC KERR EFFECT IN GaAs/AlGaAs MULTIPLE QUANTUM WELLS*
}

\author{
E. Dudziak, J. BoźYM, D. Pruchnik \\ Institute of Physics, University of Technology of Wrocław \\ Wybrzeże Wyspiańskiego 27, 50-370 Wrocław, Poland
}

AND Z.R. WASILEWSKI

Institute for Microstructural Sciences, National Research Council

K1AOR6 Ottawa, Canada

\begin{abstract}
Magnetooptic Kerr effect in $\mathrm{GaAs} / \mathrm{Al}_{0.312} \mathrm{Ga}_{0.688}$ As multiple quantum wells was investigated in the integer quantum Hall regime. The measurements have been performed in magnetic fields up to $14.5 \mathrm{~T}$, at the temperature of $1.8 \mathrm{~K}$. Experimental data indicate the discontinuous behavior of the magnetooptic Kerr effect spectrum as a function of the filling factor. For odd filling factor values $\nu=3$ and 5 we observe the large spin splitting. The effects cannot be explained in the one-particle model.
\end{abstract}

PACS numbers: 78.20.Ls, 73.20.Dx, 78.66.Fd

Interband magnetooptical spectroscopy in strong magnetic fields is a sensitive method in studies of two-dimensional electron gas (2DEG) [1-5]. It allows us to study the correlations between the optical properties of $2 \mathrm{DEG}$ and the integer and fractional quantum Hall effects (IQHE and FQHE). Many experimental and theoretical papers are devoted to quantum Hall state with filling factor $\nu=1$, when all electrons occupy the spin-split lowest Landau level [6-9]. It was established that the $\nu=1$ state is governed predominantly by the ferromagnetic many-body exchange interaction (the skyrmions). Our report concerns the magnetooptical properties of the 2DEG at higher filling factors $\nu>1$.

In most cases the method of photoluminescence (PL), photoluminescence excitation (PLE), and the method of reflectance were used. The PL and PLE depend on complicated relaxation effects that take place before the electrons relax to the lowest energy state. Therefore, some people prefer to use the magnetoabsorption $[2,6,8]$.

*The measurements have been performed at the International Laboratory of High Magnetic Fields and Low Temperatures, Wrocław, Poland. 
Two years ago we undertook the measurements of the magnetorotation of the linear polarized light reflected from the samples with multiple quantum wells (MQWs) of GaAs/AlGaAs [10]. The magnetorotation has also been measured by the other authors in InGaAs/GaAs [11] and $\mathrm{CdTe} / \mathrm{CdMnTe}$ quantum well structures [11-13].

The polarization of both transmitted and reflected linearly polarized light, propagating parallel to the external magnetic field, is in general elliptical. The angle between the major axis and the incident polarization direction defines the Faraday rotation (FR) in a transmission and the magnetooptic Kerr rotation (KR) in a reflection. Both effects can be phenomenologically described by the off-diagonal $\widetilde{\varepsilon}_{x y}$ component of the dielectric tensor in the presence of a longitudinal magnetic field: $\widetilde{\varepsilon}_{x y}=\Delta \widetilde{\varepsilon} / 2$ with $\Delta \widetilde{\varepsilon}=\widetilde{\varepsilon}^{-}-\widetilde{\varepsilon}^{+}$, where $\widetilde{\varepsilon}^{\mp}$ are the complex dielectric functions for right and left circularly polarized light $\left(\sigma^{\mp}\right)$ [14]. The angles of the FR and $\mathrm{KR}$ can be calculated from the formulas: $\phi_{\mathrm{F}}=(1 / 2)(\omega / c)\left(n^{-}-n^{+}\right) d$ and $\phi_{\mathrm{K}} \approx\left(k^{-}-k^{+}\right) /\left(n^{2}-1\right)$ if $k \ll n$, where $n, k$ are the real and imaginary parts of the complex refractive index $\tilde{n}, d$ is the sample thickness and the other symbols have the usual meanings.

When the sample is a thin film or has a multilayer structure, like in MQWs, the multiple internal reflections cause the superposition of the FR and KR in the reflection configuration and we can talk about some effective magnetorotation (MR). From our matrix calculations it results that in the dispersive spectral region of the interband magnetotransitions in QWs the magnetorotation is governed predominantly by the difference of absorption coefficients $\Delta k=k^{-}-k^{+}$. Manfra et al. [8] have shown experimentally, at least for states around $\nu=1$, that the integrated absorption peak in each polarization is proportional to the available density of states $N_{A \uparrow(\downarrow)}$ in the spin-up (down) band of the lowest Landau level. Because the Kerr rotation $\phi_{\mathrm{K}}$ results from the difference of the absorption for $\sigma^{-}$and $\sigma^{+}$polarizations, Kerr rotation $\phi_{\mathrm{K}}$ will be the measure of the difference $N_{A \downarrow}-N_{A \uparrow}$ and finally the measure of the spin polarization per particle $S_{z}=\left(N_{\uparrow}-N_{\downarrow}\right) / N=\left(N_{A \downarrow}-N_{A \uparrow}\right) / N\left(N_{\uparrow(\downarrow)}\right.$ is the number of spin-up (down) electrons in the Landau level).

Kerr rotation measurement is therefore sensitive to the magnetization measurement.

The MQWs were grown by molecular beam epitaxy (MBE). Two samples with a little different quantum well thicknesses $(183 \AA$ and $197 \AA)$ and different $n$-doping levels $\left(3.61 \times 10^{11} \mathrm{~cm}^{-2}\right.$ and $8.7 \times 10^{11} \mathrm{~cm}^{-2}$, respectively) have been measured. The barriers were asymmetrically $\delta$-doped with $\mathrm{Si}$ atoms at the distance $75 \AA$ from one side of the barriers.

The measurements were performed in the reflection configuration, at the temperature of $1.8 \mathrm{~K}$. The magnetic field (up to $14.5 \mathrm{~T}$ ) of superconductivity coil was perpendicular to the interfaces of the MQWs. Linearly polarized monochromatic light was incident normally to the sample. The polarization state of the light reflected from the sample was analysed by the rotating polarizer placed before the detector [15]. Figure 1a shows the measured magnetorotation $\Theta(E)$ spectra of sample 1 at different magnetic fields. A rich structure of magnetoexcitons was seen in a wide energy range up to the transitions to the third subband in the 

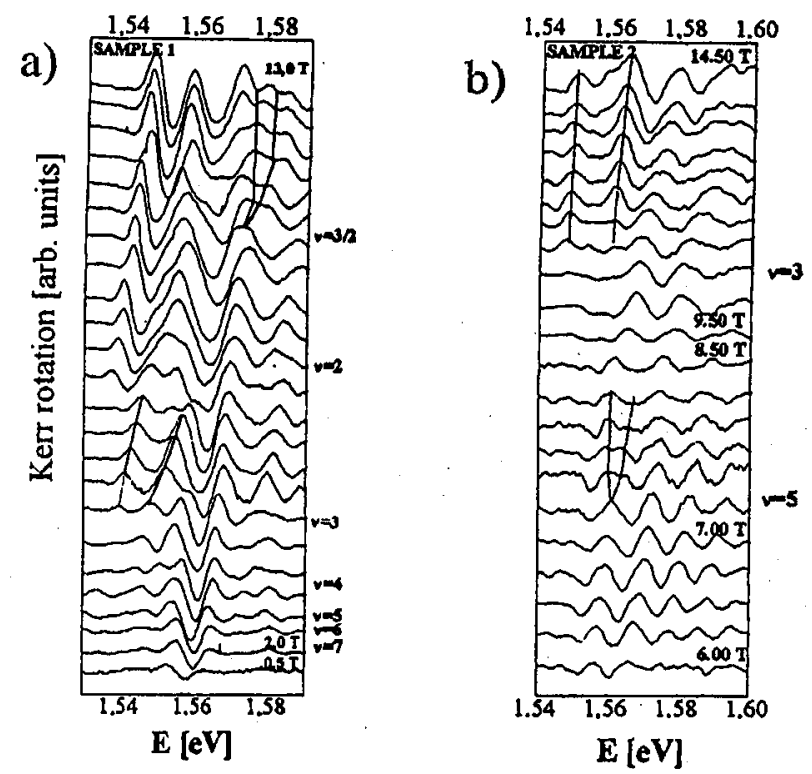

Fig. 1. Kerr rotation spectra for sample 1 (a) and sample 2 (b) measured in the quantum Hall regime (temperature $1.8 \mathrm{~K}$ and magnetic fields up to $14.5 \mathrm{~T}$ ). Dashed lines represent splittings of the transition energy which start at the odd filling factors $\nu=3$ and 5 and at the filling factor $\nu=3 / 2$.

quantum well (Fig. 1 shows only the narrow region of energies close to the occupied Landau level). A poorer and more broadened structure can be observed in sample 2 with a much higher carrier concentration (Fig. 1b).

The transition energies, estimated from $\Theta(E)$ spectra versus magnetic field (the so-called Landau fan charts) are plotted in Fig. 2. The experimental fan charts except for the lowest Landau levels populated by electrons correspond roughly to the theoretical results obtained in the single-particle approximation with a very simplified approach. In this approach the single particle states are $|m, n, \sigma\rangle$ with energies $E_{m, n, \sigma}=\hbar \omega_{\mathrm{c}}(n+1 / 2)+g \mu_{\mathrm{B}} B \sigma$, where $n$ labels Landau levels, and $m$ labels degenerate intra-Landau level states. $\hbar \omega_{c}$ is cyclotron energy.

The zero-magnetic field energy levels of the confined electrons and holes were calculated using the local density approximation (LDA) which means that the Schrödinger equation and the Poisson equation were solved self-consistently, taking account of: confining potentials, the Hartree potential of electrons and ionized donors, and exchange-correlation potential. The third step of the approach was the correction for the exciton binding energy. The exciton binding energy $E_{\mathrm{B}}$ was calculated using a modified version of the $2 \mathrm{D}$ hydrogen model described in Ref. [16].

The experimental fan charts for the lowest Landau level occupied by electrons cannot be explained in the frame of single-particle approximation. This part of the fan charts shows doublet-like splitting at $\nu=3$ and 5 . Our experimental results show a striking resemblance to the theoretical results obtained recently by Hawry- 


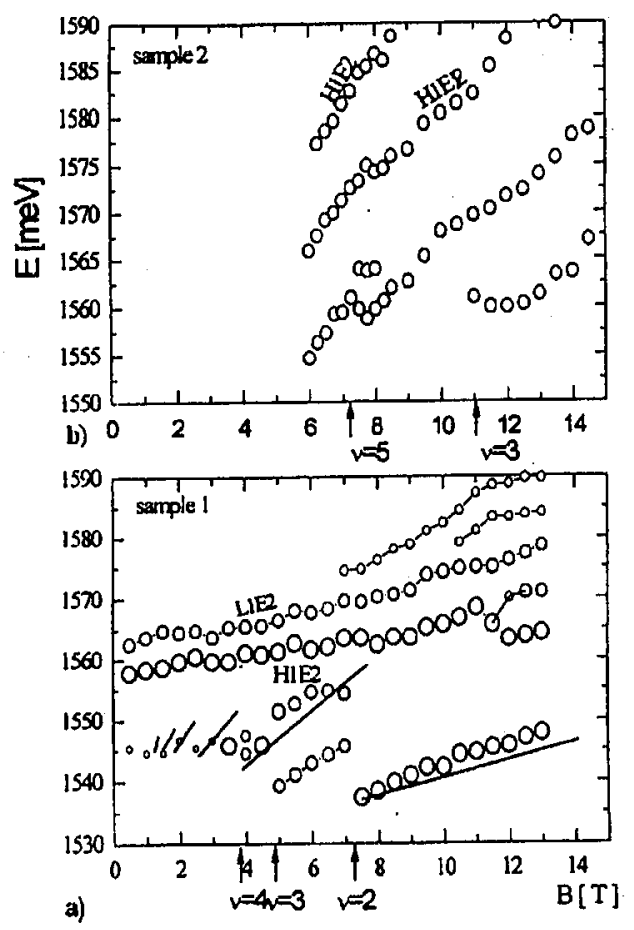

Fig. 2. The dependence of interband transition energy on the magnetic field for sample 1 (a) and 2 (b). Solid lines represent Fermi levels oscillations in the single-particle model.

lak and Potemski [17] for photoluminescence from an interacting two-dimensional gas in strong magnetic fields. They started from Hartree-Fock ground states and treat electron-electron and electron-hole interactions first. The doublet-like splitting results in a similar way as it was in the case of the Fano-Anderson problem of a localized state (hole state) in a continuum of states of band electrons. Gravier et.al. [18] have shown that for the disk with $N=48$ electrons (simulating sample), when the Coulomb interaction between the optically created hole and electrons is included, the spectral function of the hole splits into two peaks of almost equal weight.

In the interband absorption process, in the stationary state, recombination process must also exist although in much smaller scale of intensity. 'Therefore in magnetooptic Kerr effect a similar doublet-like splitting of transition energy, shifted to higher values by the Fermi energy, should also occur. Roughly speaking, for odd filling factors there is the spin splitting due to finite magnetization of the 2DEG. It seems that this splitting observed in our experiment is more distinguished than in the PL [18]. The order of magnitude of the splitting energy in our measurements agrees with the results for the PL. The spin splitting at $\nu=3$ is huge in comparison to Zeeman splitting. 
The extension of the theory to the magnetoabsorption and Kerr rotation is necessary.

In sample 1 we also observed some splitting which starts at magnetic field $10 \mathrm{~T}$ corresponding a filling factor $\nu=3 / 2$. The $\nu=3 / 2$ state is different from the IQHE states and retains its minimum value of $\rho_{x x} \neq 0$ surprisingly up to $T \approx 10 \mathrm{~K}$ at which all features of the FQHE have completely disappeared and only structures of IQHE remain [19]. Our experimental data are not sufficient to be sure that this is the only one explanation. So we cannot exclude that the observed effect is an incidental coincidence and the splitting may result for instance from the crossing of Landau levels in a complicated structure of the valence band.

In conclusion, we have shown that the KR measurements are a simple and sensitive (differential) method of investigation of the interacting 2DEG in the quantum Hall regime. Large splittings associated with odd filling factors $\nu=3$ and 5 have been found. There exists a striking resemblance to the analogous effects in the PL, but the extension of the theory to the magnetoabsorption is necessary.

We gratefully acknowledge M. Załuziny for introducing us to the LDA calculation technique. We thank Pawel Hawrylak for helpful and critical discussion.

\section{References}

[1] J.C. Maan, G. Belle, A. Fasolino, M. Altarelli, K. Ploog, Phys. Rev. B 30, 2253 (1984).

[2] B.B. Goldberg, D. Heiman, M.J. Graf, D.A. Broido, A. Pinczuk, C.W. Tu, J.H. English, A.C. Gossard, Phys. Rev. B 38, 10131 (1988).

[3] L. Viña, G.E.W. Bauer, M. Potemski, J.C. Maan, E.E. Mendez, W.I. Wang, Phys. Rev. B 41, 10767 (1990).

[4] R. Stępniewski, M. Potemski, H. Buhmann, D. Toet, J.C. Maan, G. Martinez, W. Knap, A. Raymond, B. Etienne, Phys. Rev. B 50, 11895 (1994).

[5] M. Kemerink, P.M. Koenraad, P.C.M. Christianen, R. von Schaijk, J.C. Maan, J.H. Walter, Phys. Rev. B 56, 4853 (1997).

[6] E.H. Aifer, B.B. Goldberg, D.A. Broido, Phys. Rev. Lett. 76, 680 (1996).

[7] I.V. Kukushkin, K. von Klitzing, K. Eberl, Phys. Rev. B 55, 10607 (1997).

[8] M.J. Manfra, B.B. Goldberg, L. Pfeiffer, K. West, Phys. Rev. B 54, R17327 (1996); also Acta Phys. Pol. A 92, 621 (1997).

[9] F. Plentz, D. Heiman, L.N. Pfeiffer, K.W. West, Phys. Rev. B 57, 1370 (1998).

[10] E. Dudziak, J. Bożym, D. Pruchnik, Z.R. Wasilewski, Acta Phys. Pol. A 90, 1022 (1996).

[11] A.V. Kavokin, M.R. Vladimirova, M.A. Kaliteevski, O. Lyngues, J.D. Berger, H.M. Gibbs, G. Klitrova, Phys. Rev. B 56, 1087 (1997).

[12] C. Testelin, C. Rigaux, J. Cibert, Phys. Rev. B 55, 2360 (1997).

[13] A. Lemaitre, C. Testelin, C. Rigaux, S. Maćkowski, Nguyen The Khoi, J.A. Gaj, G. Karczewski, T. Wojtowicz, J. Kossut, Phys. Rev. B 57, 4708 (1998).

[14] F.R. Kessler, J. Metzdorf, in: Landau Level Spectroscopy: Interband Effects and Faraday Rotation, Eds. G. Landwehr, E.I. Rashba, Elsevier Science Publ. North-Holland, Amsterdam 1991, Ch. 11, p. 579.

[15] J.C. Suits, Rev. Sci. Instrum. 42, 19 (1971). 
[16] A.H. Mac Donald, D.S. Ritche, Phys. Rev. B 33, 8336 (1989).

[17] P. Hawrylak, M. Potemski, Phys. Rev. B 56, 12386 (1997).

[18] L. Gravier, M. Potemski, P. Hawrylak, B. Etienne, Phys. Rev. Lett. 80, 3344 (1998).

[19] T. Chakraborty, P. Pietilainen, in: The Quantum Hall Effects, Ed. K. von Klitzing, 2nd ed., Springer-Verlag, Berlin 1995, p. 231. 